\title{
Electric dipole polarizability of alkaline-Earth-metal atoms from perturbed relativistic coupled-cluster theory with triples
}

\author{
S. Chattopadhyay, ${ }^{1}$ B. K. Mani, ${ }^{2}$ and D. Angom ${ }^{1}$ \\ ${ }^{1}$ Physical Research Laboratory, Ahmedabad - 380009, Gujarat, India \\ ${ }^{2}$ Department of Physics, University of South Florida, Tampa, Florida 33620, USA
}

\begin{abstract}
The perturbed relativistic coupled-cluster (PRCC) theory is applied to calculate the electric dipole polarizabilities of alkaline Earth metal atoms. The Dirac-Coulomb-Breit atomic Hamiltonian is used and we include the triple excitations in the relativistic coupled-cluster (RCC) theory. The theoretical issues related to the triple excitation cluster operators are described in detail and we also provide details on the computational implementation. The PRCC theory results are in good agreement with the experimental and previous theoretical results. We, then, highlight the importance of considering the Breit interaction for alkaline Earth metal atoms.

PACS numbers: 31.15.bw,31.15.ap,31.15.A-,31.15.ve
\end{abstract}

\section{INTRODUCTION}

Static electric dipole polarizability, $\alpha$ of the neutral alkaline Earth metal atoms [1] is important for many applications in the ongoing and future experiments. To name a few, the parity and time reversal violation in atoms [2, 3], optical atomic clocks $[4,5]$ and condensates of dilute atomic gases [6-8] are of current interest. In the past different many-body methods have been used to calculate $\alpha$ precisely. Some of the theoretical methods used to calculate $\alpha$ are described in a recent review by Mitroy et. al [9]. Another important and excellent reference for the ground state $\alpha$ of neutral atoms is the table of experimental data and theoretical results prepared by Schwerdtfeger [10]. In this paper we employ the perturbed relativistic coupled-cluster (PRCC) theory $[11,12]$ to calculate the $\alpha$ of alkaline Earth metal atoms. The theoretical details of PRCC theory are discussed in our previous works [11-14]. A related method used for calculating electric dipole polarizabilities is to consider only the $z$-component of the dipole operator and define a set of perturbed cluster operators [15]. In PRCC theory we introduce a new set of cluster operators along with the original RCC operators. For convenient description we refer to the latter as the unperturbed cluster operators. The technique is general and suitable to incorporate multiple perturbations for structure and properties calculations of many electron atoms and ions. So, in the PRCC theory, the cluster operators can be scalar or tensor operators of any rank and this is an important feature of the PRCC theory we have developed.

Among the different many body techniques the coupled-cluster theory (CCT) based methods are efficient and powerful. A recent review [16] on the CCT gives an insight about the theory and related CCT based techniques. The CCT has been widely used for atomic [1721], molecular [22], nuclear [23] and condensed matter physics [24] calculations. In the present work, we introduce the triple excitations in the RCC theory to go beyond coupled-cluster single and double (CCSD) approximation. The inclusion of the triple excitations incorpo- rate some of the many-body effects equivalent to the diagrams which begin to contribute from the second order in many-body perturbation theory (MBPT). The triple excitations at the RCC theory shall pave the way for high precision results for atomic structure calculations. It must be mentioned that, a previous work have considered the triple excitation cluster amplitudes in RCC calculations [25]. In this work we, however, introduce a different but equivalent representation of the triple excitation cluster operator. So, in the present work, the unperturbed clusters are calculated with the relativistic coupled-cluster single, double and triple (RCCSDT) excitation approximation. We, however, use the PRCC theory with single and double excitation approximation. In our future work we shall include the effects of triple excitations in the PRCC theory.

The paper is organized as follows. In the Sec. II. A, we briefly introduce the RCC and PRCC theories with the Dirac-Coulomb-Breit Hamiltonian. In Sec. II. B, we introduce the triple excitations $\left(T_{3}^{(0)}\right)$ in the RCC theory and derive the RCCSDT amplitude equations. In the next part we introduce the angular momentum diagrams corresponding to $T_{3}^{(0)}$ operator and evaluation of $T_{3}^{(0)}$ diagrams in the RCC theory. In Sec. III we briefly discuss about the $\alpha$ calculations in the framework of PRCC theory. In the subsequent sections we describe the calculational details and the computational issues related to $T_{3}^{(0)}$ calculations. We, then, present the results and discussions for neutral alkaline Earth metal atoms and end with conclusions. All the results presented in this work and related calculations are in atomic units $\left(\hbar=m_{e}=e=1 / 4 \pi \epsilon_{0}=1\right)$. In this system of units the velocity of light is $\alpha^{-1}$, the inverse of fine structure constant. For which we use the value of $\alpha^{-1}=137.035999074$ [26].

\section{THEORETICAL METHODS}

For the high- $Z$ atoms and ions, the Dirac-CoulombBreit Hamiltonian, denoted by $H^{\mathrm{DCB}}$, is an appropri- 
ate choice to include the relativistic effects. However, there are complications associated with the negative energy continuum states of $H^{\mathrm{DCB}}$. These lead to variational collapse and continuum dissolution [27]. A formal approach to avoid these complications is to use the novirtual-pair approximation. In this approximation, for a neutral atom of $N$ electrons [28]

$$
\begin{aligned}
H^{\mathrm{DCB}}= & \Lambda_{++} \sum_{i=1}^{N}\left[c \boldsymbol{\alpha}_{i} \cdot \mathbf{p}_{i}+\left(\beta_{i}-1\right) c^{2}-V_{N}\left(r_{i}\right)\right] \\
& +\sum_{i<j}\left[\frac{1}{r_{i j}}+g^{\mathrm{B}}\left(r_{i j}\right)\right] \Lambda_{++},
\end{aligned}
$$

where $\boldsymbol{\alpha}$ and $\beta$ are the Dirac matrices, $\Lambda_{++}$is an operator which projects to the positive energy solutions and $V_{N}\left(r_{i}\right)$ is the nuclear potential. Sandwiching the Hamiltonian with $\Lambda_{++}$ensures that the effects of the negative energy continuum states are neglected in the calculations. Another approach, which is better suited for numerical computations, is to use the kinetically balanced finite basis sets [29-32]. We use this method in the present work to generate the orbital basis sets. The last two terms, $1 / r_{i j}$ and $g^{\mathrm{B}}\left(r_{i j}\right)$ are the Coulomb and Breit interactions, respectively. The latter, Breit interaction, represents the inter-electron magnetic interactions and is given by

$$
g^{\mathrm{B}}\left(r_{12}\right)=-\frac{1}{2 r_{12}}\left[\boldsymbol{\alpha}_{1} \cdot \boldsymbol{\alpha}_{2}+\frac{\left(\boldsymbol{\alpha}_{\mathbf{1}} \cdot \mathbf{r}_{12}\right)\left(\boldsymbol{\alpha}_{\mathbf{2}} \cdot \mathbf{r}_{12}\right)}{r_{12}^{2}}\right] .
$$

The Hamiltonian satisfies the eigen-value equation

$$
H^{\mathrm{DCB}}\left|\Psi_{i}\right\rangle=E_{i}\left|\Psi_{i}\right\rangle
$$

where, $\left|\Psi_{i}\right\rangle$ is the exact atomic state and $E_{i}$ is the energy of the atomic state. In the presence of external electromagnetic fields, the Hamiltonian is modified with the addition of interaction terms. For external static electric field, the interaction is $H_{\text {int }}=-\mathbf{d} \cdot \mathbf{E}_{\text {ext }}$, where $\mathbf{d}$ and $\mathbf{E}_{\text {ext }}$ are the induced electric dipole moment of the atom and external electric field, respectively. In the remaining part of this section we give a brief description of RCC theory, which we use to compute atomic state $|\Psi\rangle$ and PRCC to account for the effects $H_{\text {int }}$ in the atomic state.

\section{A. RCC and PRCC theories}

In RCC theory we define the ground state atomic wavefunction of a closed-shell atom as

$$
\left|\Psi_{0}\right\rangle=e^{T^{(0)}}\left|\Phi_{0}\right\rangle
$$

where $\left|\Phi_{0}\right\rangle$ is the reference state wave-function and $T^{(0)}$ is the unperturbed cluster operator. To account for the correction to the wavefunction arising from $H_{\text {int }}$, we define the perturbed ground state as

$$
\left|\tilde{\Psi}_{0}\right\rangle=e^{T^{(0)}+\lambda \mathbf{T}^{(1)} \cdot \mathbf{E}}\left|\Phi_{0}\right\rangle=e^{T^{(0)}}\left[1+\lambda \mathbf{T}^{(\mathbf{1})} \cdot \mathbf{E}\right]\left|\Phi_{0}\right\rangle,
$$

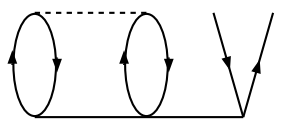

(a)

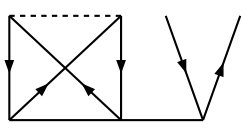

(b)
FIG. 1. Diagrams of $T_{1}^{(0)}$ cluster operator arising from the triple excitation cluster operator, $T_{3}^{(0)}$.

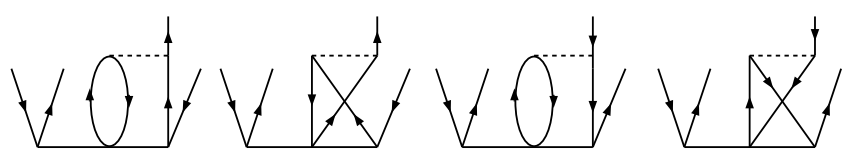

(a) (b) (c)

(d)
FIG. 2. Diagrams of $T_{2}^{(0)}$ cluster operator arising from the triple excitation cluster operator, $T_{3}^{(0)}$.

where $\mathbf{T}^{(1)}$ are the PRCC operators $[11,12]$. For an $N$ electron closed-shell atom $T^{(0)}=\sum_{i=1}^{N} T_{i}^{(0)}$ and $\mathbf{T}^{(1)}=$ $\sum_{i=1}^{N} \mathbf{T}_{i}^{(1)}$, where $i$ is the order of excitation. In the coupled-cluster single and double (CCSD) excitation approximation [33],

$$
\begin{aligned}
& T^{(0)}=T_{1}^{(0)}+T_{2}^{(0)}, \\
& \mathbf{T}^{(1)}=\mathbf{T}_{1}^{(1)}+\mathbf{T}_{2}^{(1)} .
\end{aligned}
$$

The CCSD is a good starting point for structure and properties calculations of closed-shell atoms and ions. In the second quantized representation

$$
\begin{aligned}
T_{1}^{(0)} & =\sum_{a, p} t_{a}^{p} a_{p}^{\dagger} a_{a}, \\
T_{2}^{(0)} & =\frac{1}{(2 !)^{2}} \sum_{a, b, p, q} t_{a b}^{p q} a_{p}^{\dagger} a_{q}^{\dagger} a_{b} a_{a} \\
\mathbf{T}_{1}^{(1)} & =\sum_{a, p} \tau_{a}^{p} \mathbf{C}_{1}(\hat{r}) a_{p}^{\dagger} a_{a}, \\
\mathbf{T}_{2}^{(1)} & =\frac{1}{(2 !)^{2}} \sum_{a, b, p, q} \sum_{l, k} \tau_{a b}^{p q}(l, k)\left\{\mathbf{C}_{l}\left(\hat{r}_{1}\right) \mathbf{C}_{k}\left(\hat{r}_{2}\right)\right\}^{1} a_{p}^{\dagger} a_{q}^{\dagger} a_{b} a_{a},
\end{aligned}
$$

where $t_{\ldots}^{\ldots}$ and $\tau_{\ldots}^{\ldots}$ are the cluster amplitudes, $a_{i}^{\dagger}\left(a_{i}\right)$ are single particle creation (annihilation) operators and $a b c \ldots(p q r \ldots)$ represent core (virtual) single particle states or orbitals. To represent $\mathbf{T}_{1}^{(1)}$, a rank one operator, we have used the $\mathbf{C}$-tensor of similar $\operatorname{rank} \mathbf{C}_{1}(\hat{r})$. Coming to $\mathbf{T}_{2}^{(1)}$, to represent it two $\mathbf{C}$-tensor operators of rank $l$ and $k$ are coupled to a rank one tensor operator. In addition, the PRCC clusters are constrained by other selection rules arising from parity and triangular conditions, these are described in our previous work [12].

\section{B. CCSDT approximation}

The RCCSD approximation encompasses a major part of the electron correlation effects. It is, however, per- 
tinent to incorporate triple excitation cluster operator, $T_{3}^{(0)}$, to obtain higher precision. With $T_{3}^{(0)}$, the theory is referred to as the relativistic coupled-cluster single, double and triple (RCCSDT) excitation approximation, then $T^{(0)}=T_{1}^{(0)}+T_{2}^{(0)}+T_{3}^{(0)}$. In the second quantized notations, we may write the triple excitation cluster operator as

$$
T_{3}^{(0)}=\frac{1}{(3 !)^{2}} \sum_{\substack{a, b, c \\ p, q, r}} t_{a b c}^{p q r} a_{p}^{\dagger} a_{q}^{\dagger} a_{r}^{\dagger} a_{c} a_{b} a_{a},
$$

and the cluster operators are the solutions of the equation

$$
\left\langle\Phi_{a b c}^{p q r}\left|\bar{H}_{\mathrm{N}}^{\mathrm{DCB}}\right| \Phi_{0}\right\rangle=0 .
$$

Where, $H_{\mathrm{N}}^{\mathrm{DCB}}$ is the normal ordered Dirac-CoulombBreit Hamiltonian and $\bar{H}_{\mathrm{N}}^{\mathrm{DCB}}=e^{-T^{(0)}} H_{\mathrm{N}}^{\mathrm{DCB}} e^{T^{(0)}}$ is the similarity transformed or dressed Hamiltonian. Here after, for compact notation we use $\bar{H}_{\mathrm{N}}$ to represent $\bar{H}_{\mathrm{N}}^{\mathrm{DCB}}$. Following Wick's theorem and structure of $H_{\mathrm{N}}$, in general

$$
\begin{aligned}
& \bar{H}_{\mathrm{N}}=H_{\mathrm{N}}+\left\{{\widehat{H_{\mathrm{N}}}}^{(0)}\right\}+\frac{1}{2 !}\left\{{\widetilde{H_{\mathrm{N}} T^{(0)}}}^{(0)}\right\}+ \\
& \frac{1}{3 !}\left\{\overline{\overline{H_{\mathrm{N}} T^{(0)}}} T^{(0)} T^{(0)}\right\}+\frac{1}{4 !}\left\{\overline{\overline{H_{\mathrm{N}} T^{(0)}} T^{(0)}} T^{(0)} T^{(0)}\right\},
\end{aligned}
$$

where $\overparen{A \ldots B}$ denotes contraction between the two operators $A$ and $B$, and $\{\cdots\}$ represent normal ordering of the operator. A detailed description of the CCSDT cluster equations, in the context of non-relativistic systems, is given the in the recent book of Shavitt and Bartlett [34]. The equations can be modified to the relativistic case. Although, we solve the full RCCSDT equations, for a more compact description but to provide all the key details, here we give the description of linearised RCCSDT (LRCCSDT). In this approximation we retain terms in the $T^{(0)}$ equations which are zeroth and first order in $T^{(0)}$. It is an approximation which is relatively simple but incorporates all the important many-body effects. In addition, solving the LRCCSDT equations is not computationally intensive, for this reason, we solve these equations first and use the results as starting values to solve the full or nonlinear RCCSDT equations. In the LRCCSDT the cluster amplitude equations are then

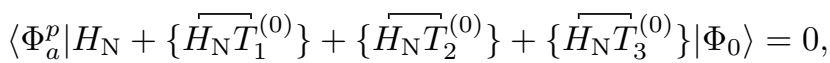

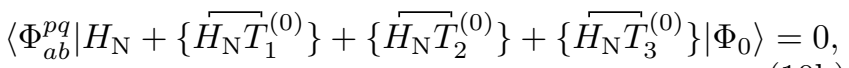

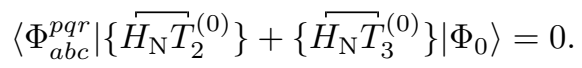

Here, an important observation is the absence of $H_{\mathrm{N}}$ and $\left\{\bar{H}_{\mathrm{N}} T_{1}^{(0)}\right\}$ in the $T_{3}^{(0)}$ equation. The reason is, as $H_{\mathrm{N}}$ is a two-body interaction Hamiltonian, at first order it does not induce triple excitations by itself or after contraction with $T_{1}^{(0)}$. In the LRCCSDT, $T_{1}^{(0)}$ and $T_{2}^{(0)}$ have, respectively, two and four diagrams arising from from $T_{3}^{(0)}$. The latter are shown in Fig. 2 and these originate from two types of residual two-body interactions, namely $g_{p q}^{r a}$ and $g_{a p}^{b c}$, where $g_{i j}^{k l}=\left\langle k l\left|1 / r_{12}+g^{B}\left(r_{12}\right)\right| i j\right\rangle$. In Fig. 2, the diagrams (a-b) and (c-d) arise from $g_{p q}^{r a}$ and $g_{a p}^{b c}$ respectively. Similarly, the diagrams of $T_{3}^{(0)}$ arising from $T_{3}^{(0)}$ in the linearized RCCSDT are shown in Fig. 3. There are eight diagrams and these arise from six types of residual Coulomb interactions $g_{p a}^{q r}, g_{a b}^{c p}, g_{a q}^{p b}, g_{p a}^{q b}, g_{p q}^{r s}$ and $g_{a b}^{c d}$. The contribution from $T_{3}^{(0)}$ to the $T_{1}^{(0)}$ cluster equation, Eq. (10a), may be written in the algebraic form as

$$
\left\langle\widehat{H}_{\mathrm{N}} T_{3}^{(0)}\right\rangle_{a}^{p}=\sum_{b c q r}\left(g_{q r}^{b c}-g_{r q}^{b c}\right) t_{a b c}^{p q r}
$$

where $\langle\cdots\rangle_{a}^{p}$ represents $\left\langle\Phi_{a}^{p}|\cdots| \Phi_{0}\right\rangle$. Similarly, the $T_{3}^{(0)}$ contribution to the $T_{2}^{(0)}$ cluster operator equation, fourth term in Eq. (10b), is

$$
\left\langle{\overrightarrow{H_{\mathrm{N}} T_{3}}}^{(0)}\right\rangle_{a b}^{p q}=\sum_{r c s}\left(g_{c q}^{r s}-g_{c q}^{s r}\right) t_{a c b}^{p r s}+\frac{1}{2} \sum_{r c d}\left(g_{c d}^{r b}-g_{d c}^{r b}\right) t_{a c d}^{p r q} .
$$

With these definitions of the terms arising from $T_{3}^{(0)}$, the $T_{1}^{(0)}$ cluster amplitude equation, Eq. (10a), in algebraic form is

$$
\begin{aligned}
& \sum_{b q} g_{a q}^{b p} t_{b}^{q}+\frac{1}{2} \sum_{b c q} g_{q a}^{b c}\left(t_{b c}^{q p}-t_{b c}^{p q}\right)+\sum_{b q r} g_{q r}^{b p}\left(t_{b a}^{q r}-t_{a b}^{q r}\right) \\
& +\frac{1}{2} \sum_{b c q r}\left(g_{q r}^{b c}-g_{r q}^{b c}\right) t_{a b c}^{p q r}+\left(\varepsilon_{p}-\varepsilon_{a}\right) t_{a}^{p}=0
\end{aligned}
$$

where, $\varepsilon_{i}$ is the orbital energy of the $i$ th orbital. Similarly, the $T_{2}^{(0)}$ cluster amplitude equation, Eq. (10b), can be written in algebraic form as

$$
\begin{aligned}
& \sum_{r} g_{a r}^{p q} t_{b}^{r}-\sum_{c} g_{a b}^{p c} t_{c}^{q}+\sum_{c d} g_{a b}^{c d} t_{c d}^{p q}+\sum_{r s} g_{r s}^{p q} t_{a b}^{r s}-\sum_{c r}\left[g_{a r}^{c p} t_{c b}^{r q}\right. \\
& \left.+g_{r b}^{p c} r_{a c}^{r q}+\frac{1}{2} g_{a r}^{p c}\left(t_{c b}^{r q}-t_{b c}^{r q}\right)\right]+\sum_{r c s}\left(g_{c q}^{r s}-g_{c q}^{s r}\right) t_{a c b}^{p r s}+\frac{1}{2} \sum_{r c d}\left(g_{c d}^{r b}\right. \\
& \left.-g_{d c}^{r b}\right) t_{a c d}^{p r q}+\left(\begin{array}{c}
p \leftrightarrow q \\
a \leftrightarrow c
\end{array}\right)+\left(\varepsilon_{p}+\varepsilon_{q}-\varepsilon_{a}-\varepsilon_{b}\right) t_{a b}^{p q}=0,
\end{aligned}
$$

where $i \leftrightarrow j$ represents permutation of the two indexes. As evident from the $T_{3}^{(0)}$ cluster equation, Eq. (10c), the contributing terms are the contraction of $H_{\mathrm{N}}$ with $T_{2}^{(0)}$ and $T_{3}^{(0)}$. The Goldstone diagrams arising from these terms are shown in Fig. 3, where the diagrams in Fig. $3(\mathrm{a}-\mathrm{b})$ arise from $T_{2}^{(0)}$, and the diagrams in Fig. 3(c-h) arise from $T_{3}^{(0)}$. Collecting all the diagrams, the equation of the $T_{3}^{(0)}$ cluster amplitude in algebraic form is

$$
\begin{aligned}
& \sum_{s} g_{s c}^{q r} t_{a b}^{p s}+\sum_{d} g_{b c}^{d r} t_{a d}^{p q}+\sum_{d s}\left[g_{p d}^{a s}\left(t_{d b c}^{s q r}+t_{b d c}^{s q r}\right)+g_{p d}^{s b} t_{a d c}^{s d r}\right. \\
& \left.+g_{d p}^{a s} t_{d b c}^{s q r}\right]+\sum_{s t} g_{p q}^{s t} t_{a b c}^{s t r}+\sum_{d e} g_{d e}^{a b} t_{d e c}^{p q r}+\left(\begin{array}{c}
p \leftrightarrow q \leftrightarrow r \\
a \leftrightarrow b \leftrightarrow c
\end{array}\right) \\
& +\left(\varepsilon_{p}+\varepsilon_{q}+\varepsilon_{r}-\varepsilon_{a}-\varepsilon_{b}-\varepsilon_{c}\right) t_{a b c}^{p q r}=0 .
\end{aligned}
$$


With the inclusion of $T_{3}^{(0)}$ the RCC theory incorporates all the correlation effects up to second order in the residual Coulomb interaction. That is, the theory encapsulates all the many-body perturbation theory (MBPT) diagrams [35] which are first and second order in the residual Coulomb interaction. In addition, as it is coupled cluster theory, it incorporates the connected single, double and triple excitations to all order. The leading order contribution to the uncertainty in the calculations arise from the quadruple excitations, which, in MBPT, first appear at the third order of perturbation.

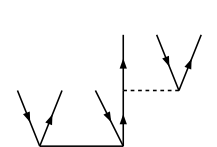

(a)

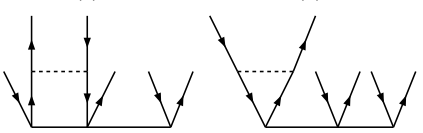

(e)

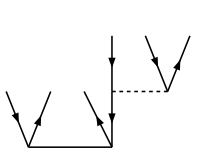

(b)

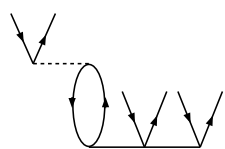

(c)

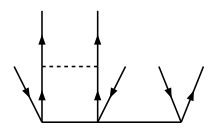

(g)

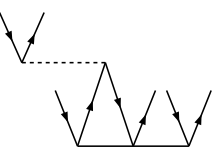

(d)

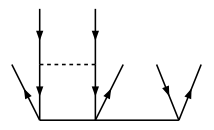

(h)
FIG. 3. Diagrams of $T_{3}^{(0)}$ arising from $T_{3}^{(0)}$ in the linearized RCCSDT theory.

\section{Representation of $T_{3}^{(0)}$}

To evaluate angular factors it is essential to employ a diagrammatic representation which is consistent with the angular momentum coupling sequence. Following the conventions of diagrammatic representation of angular momentum coupling in ref. [35], the diagram in Fig. 4 is the equivalent angular momentum diagram of the $T_{3}^{(0)}$ . This is, however, not the only representation possible,

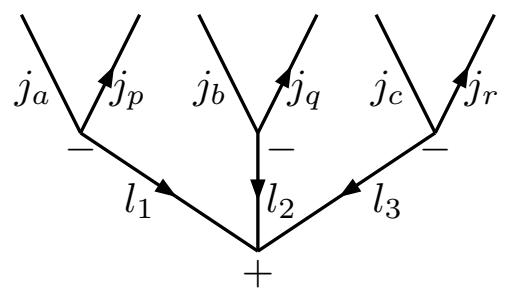

FIG. 4. Angular momentum representation of $T_{3}^{(0)}$ cluster operator. The $-(+)$ sign indicates the angular momenta at the vertices are coupled in clock (anti-clock) wise sequence. An arrow, pointing away from the vertex, on a line with angular momentum $j_{i}$ represents a phase factor of $(-1)^{j_{i}-m_{i}}$.

there is another equivalent and elegant representation described in ref. [36]. Except for the topology, the two representations require the same number of multipoles and should give the same results.

In the representation we have used the diagram is symmetric or invariant under the permutation of the vertices. There are four vertices in the angular momentum diagram, out of which three involve coupling of angular momentum of the spin-orbitals and these are $\left(j_{a}, j_{p}, l_{1}\right)$, $\left(j_{b}, j_{q}, l_{2}\right)$ and $\left(j_{c}, j_{r}, l_{3}\right)$. The last one, $\left(l_{1}, l_{2}, l_{3}\right)$, involves coupling of the multipoles associated with the orbital vertices. Following the angular momentum coupling, the vertices must satisfy the triangular conditions $\left|j_{a}-j_{p}\right| \leqslant l_{1} \leqslant\left(j_{a}+j_{p}\right),\left|j_{b}-j_{q}\right| \leqslant l_{2} \leqslant\left(j_{b}+j_{q}\right),\left|j_{c}-j_{r}\right| \leqslant$ $l_{3} \leqslant\left(j_{c}+j_{r}\right)$ and $\left|l_{1}-l_{2}\right| \leqslant l_{3} \leqslant\left(l_{1}+l_{2}\right)$. Similarly, from the parity considerations, the orbital angular momenta must satisfy the condition that $l_{a}+l_{b}+l_{c}+l_{p}+l_{q}+l_{r}$ is even.

\section{DIPOLE POLARIZABILITY}

From the second order time-independent perturbation theory, the ground state dipole polarizability of a closedshell atom is

$$
\alpha=-2 \sum_{I} \frac{\left\langle\Psi_{0}|\mathbf{D}| \Psi_{I}\right\rangle\left\langle\Psi_{I}|\mathbf{D}| \Psi_{0}\right\rangle}{E_{0}-E_{I}},
$$

where $\left|\Psi_{I}\right\rangle$ are the intermediate atomic states and $E_{I}$ is the energy of the atomic state. As $\mathbf{D}$ is an odd parity operator, $\left|\Psi_{I}\right\rangle$ must be opposite in parity to $\left|\Psi_{0}\right\rangle$. For calculations with the RCCSDT wavefunction, there is a subtle issue depending on how the $T^{(0)}$ equations are implemented and solved. By definition, following the linked-cluster theorem, RCCSDT state $\left|\Psi_{0}\right\rangle$ consists of only linked and exclusion principle obeying (EPO) diagrams. So, in the computational implementations of the RCCSDT method, one must ensure that only EPO diagrams are evaluated and included in the equations. The electric dipole polarizability in terms of the PRCC wavefunction is then

$$
\alpha=-\left(\left\langle\tilde{\Psi}_{0}|\mathbf{D}| \tilde{\Psi}_{0}\right\rangle\right)_{\text {conn }},
$$

where the subscript 'conn' represents only connected terms. In practice, however, selecting only the EPO diagrams require several conditional statements and it is computationally very inefficient. A much faster implementation is to do an unrestricted evaluation along with the exclusion principle violating (EPV) diagrams and remove the contributions at the end of the calculations. Detailed discussions on different aspects of EPV diagrams in many-body calculations are given in ref. [34]. So, in general, the terms in the RCCSDT equations are computed without restrictions on the contracted or internal orbital lines. The computed cluster amplitudes and the RCCSDT states, then, have EPV diagrams and for future reference define the corresponding RCCSDT and PRCC states as $\left|\Psi_{0}^{\prime}\right\rangle$ and $\left|\tilde{\Psi}_{0}^{\prime}\right\rangle$, respectively. However, when all possible connected diagrams are considered, we can factor the expectation of $\mathbf{D}$ in terms of the EPV states as product of 'connected' expectation part and normalization factor [37]. For the present work, we can write

$$
\left\langle\tilde{\Psi}_{0}^{\prime}|\mathbf{D}| \tilde{\Psi}_{0}^{\prime}\right\rangle=\left(\left\langle\tilde{\Psi}_{0}|\mathbf{D}| \tilde{\Psi}_{0}\right\rangle\right)_{\text {conn }}\left\langle\tilde{\Psi}_{0}^{\prime} \mid \tilde{\Psi}_{0}^{\prime}\right\rangle .
$$


With this definition, the electric dipole polarizability of a closed-shell atom in terms of the EPV coupled-cluster state is

$$
\alpha=-\frac{\left\langle\tilde{\Psi}_{0}^{\prime}|\mathbf{D}| \tilde{\Psi}_{0}^{\prime}\right\rangle}{\left\langle\tilde{\Psi}_{0}^{\prime} \mid \tilde{\Psi}_{0}^{\prime}\right\rangle}=-\left(\left\langle\tilde{\Psi}_{0}|\mathbf{D}| \tilde{\Psi}_{0}\right\rangle\right)_{\mathrm{conn}} .
$$

So, it must be mentioned that, in general, the expectation value of an operator must be normalized when the condition of EPO is not imposed while calculating the cluster amplitudes. The reason is

$$
\left(\left\langle\tilde{\Psi}_{0}^{\prime}|\mathbf{D}| \tilde{\Psi}_{0}^{\prime}\right\rangle\right)_{\text {conn }} \neq\left(\left\langle\tilde{\Psi}_{0}|\mathbf{D}| \tilde{\Psi}_{0}\right\rangle\right)_{\text {conn }},
$$

as $\left(\left\langle\tilde{\Psi}_{0}^{\prime}|\mathbf{D}| \tilde{\Psi}_{0}^{\prime}\right\rangle\right)_{\text {conn }}$, though connected, retains EPV diagrams subsumed in the definition of the cluster amplitudes. The definition of $\alpha$ in Eq. (17) is what we use in our present and previous works. Since, the implementation with EPV diagrams is the usual case, we drop the 'prime' notation here after and inclusion of EPV diagrams is implied. From the definition of $\left|\tilde{\Psi}_{0}\right\rangle$ in Eq. (5) and based on the parity selection rules, only the terms linear in $\lambda$ are nonzero. That is,

$$
\alpha=-\frac{\left\langle\Phi_{0}\left|\mathbf{T}^{(1) \dagger} \overline{\mathbf{D}}+\overline{\mathbf{D}} \mathbf{T}^{(1)}\right| \Phi_{0}\right\rangle}{\left\langle\Psi_{0} \mid \Psi_{0}\right\rangle},
$$

where, $\overline{\mathbf{D}}=e^{T^{(0) \dagger}} \mathbf{D} e^{T^{(0)}}$, represents the unitary transformed electric dipole operator and $\left\langle\Psi_{0} \mid \Psi_{0}\right\rangle$ is the normalization factor. From here on, it is implicit that expressions with more than one operator involves contraction and for compact notation, we drop the notation to represent operator contractions. Retaining the the leading order terms, we obtain

$$
\begin{aligned}
\alpha \approx & \frac{1}{\mathcal{N}}\left\langle\Phi_{0}\right| \mathbf{T}_{1}^{(1) \dagger} \mathbf{D}+\mathbf{D} \mathbf{T}_{1}^{(1)}+\mathbf{T}_{1}^{(1) \dagger} \mathbf{D} T_{1}^{(0)}+T_{1}^{(0) \dagger} \mathbf{D} \mathbf{T}_{1}^{(1)} \\
& +\mathbf{T}_{2}^{(1) \dagger} \mathbf{D} T_{1}^{(0)}+T_{1}^{(0) \dagger} \mathbf{D} \mathbf{T}_{2}^{(1)}+\mathbf{T}_{1}^{(1) \dagger} \mathbf{D} T_{2}^{(0)} \\
& +T_{2}^{(0) \dagger} \mathbf{D} \mathbf{T}_{1}^{(1)}+\mathbf{T}_{2}^{(1) \dagger} \mathbf{D} T_{2}^{(0)}+T_{2}^{(0) \dagger} \mathbf{D} \mathbf{T}_{2}^{(1)}\left|\Phi_{0}\right\rangle, \quad(20)
\end{aligned}
$$

where $\mathcal{N}=\left\langle\Phi_{0}\left|\exp \left[T^{(0) \dagger}\right] \exp \left[T^{(0)}\right]\right| \Phi_{0}\right\rangle$ is the normalization factor, which involves a non-terminating series of contractions between $T^{(0)^{\dagger}}$ and $T^{(0)}$. However, in the present work we use $\mathcal{N} \approx\left\langle\Phi_{0}\left|T_{1}^{(0) \dagger} T_{1}^{(0)}+T_{2}^{(0) \dagger} T_{2}^{(0)}\right| \Phi_{0}\right\rangle$. From the above expression of $\alpha$, an evident advantage of calculation using PRCC theory is the absence of summation over $\left|\Psi_{I}\right\rangle$. The summation is subsumed in the evaluation of the $\mathbf{T}^{(1)}$ in a natural way. This is one of the key advantage of using PRCC theory.

To incorporate the leading order contribution from $T_{3}^{(0)}$, there are two possible combinations in the PRCC expression $\alpha$. These are, $T_{3}^{(0)^{\dagger}} \mathbf{D} \mathbf{T}_{2}^{(1)}$ and hermitian conjugate. The diagrams which arise from this term, $T_{3}^{(0) \dagger} \mathbf{D} \mathbf{T}_{2}^{(1)}$, are shown in Fig. 5. In the the present work we incorporate these four diagrams to study the contribution from $T_{3}^{(0)}$ in the calculation of $\alpha$. To summarize this section, we consider all the terms that will arise at the CCSD level and along with that we consider the diagrams from the contraction of $T_{3}^{(0) \dagger} \mathbf{D} \mathbf{T}_{2}^{(1)}$.

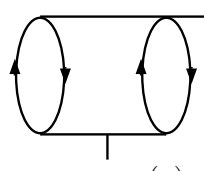

(a)

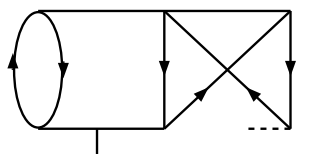

(c)

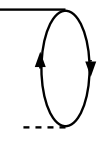

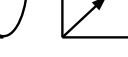

1

(b)

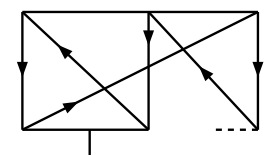

(d)
FIG. 5. Diagrams of electric dipole polarizability $\alpha$ arising from the term $T_{3}^{(0) \dagger} \mathbf{D} \mathbf{T}_{2}^{(1)}$, the is the dominant contribution with triple excitation.

\section{COMPUTATIONAL DETAILS}

\section{A. Angular integration of diagrams with $T_{3}^{(0)}$}

In this section we discuss the angular integration of the diagrams arising from $T_{3}^{(0)}$, shown in Fig. 1, 2 and 3 , in the cluster equations. For this we resort to angular momentum diagrams and follow the conventions of Lindgren and Morrison [35]. We discuss three examples to describe the notations and conventions we have adopted for angular integration. These examples, in particular, illustrate the incorporation of the $T_{3}^{(0)}$ diagrams in the angular momentum diagram evaluation. As first example let us consider a relatively simple case, the Goldstone diagram in Fig. 1(a). It is a direct interaction diagram, and evaluation does not involve any $3 j$ - or $6 j$ - symbols. The corresponding angular momentum diagram is shown in Fig. 6 , where $j_{a}, j_{b}, j_{c}\left(j_{p}, j_{q}, j_{r}\right)$ are the total angular

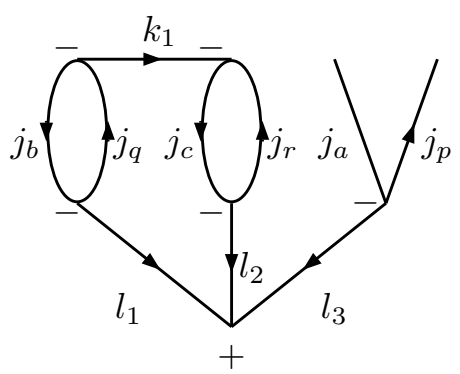

FIG. 6. Angular part of the diagram in Fig. 1(a) represented as angular momentum diagram. The diagram is evaluated using standard angular momentum coupling and summation identities represented as diagrams.

momentum of the core (virtual) orbitals, $k_{1}$ represents the multipole of the two electron Coulomb-Breit interaction and $l_{i}$ are the multipole lines associated with $T_{3}^{(0)}$. To simplify and evaluate the diagram we use angular momentum diagram identities like bubble removal, and JLV 
theorems [35]. After simplification, the diagram in Fig. 6 is equivalent to the result shown in Fig. 7. As can

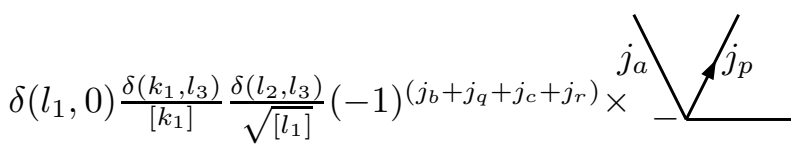

FIG. 7. Expression obtained after angular integration, using diagrammatic techniques, of the diagram in Fig. 6.

be seen from the figure, the result consists of Kronecker delta function, phase factor and angular momentum diagram of the free orbital lines of the $T_{1}^{(0)}$ operator. Algebraically, the remnant angular momentum diagram or the free part in Fig. 7 is equivalent to the geometric part in the matrix element defined using the Wigner-Eckart theorem, which explicitly depends on the magnetic quantum numbers of the initial and final states. The free part, however, is common to all the terms and to compute $T^{(0)}$ amplitudes, we define the RCC equations in terms of the reduced matrix elements.

As the second example, consider the exchange diagram of Fig. 1(a) as shown in Fig. 1(b). The corresponding angular momentum diagram is given in Fig. 8. Here, we must mention that, in general, the angular momentum diagrams of the exchange interaction diagrmas are topologically more intricate than the direct interaction diagrams. For this diagram, after simplification, we obtain

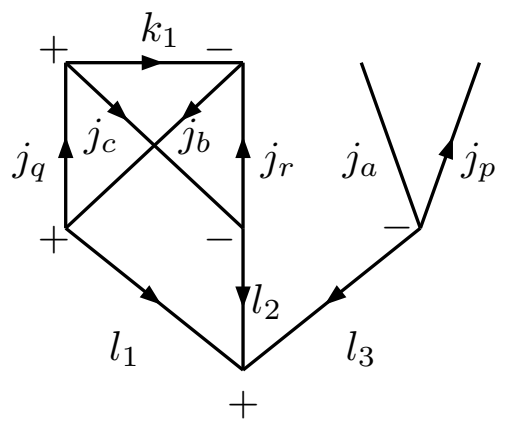

FIG. 8. Angular momentum diagram corresponding to the Goldstone diagram in Fig. 1(b). This is a diagram with exchange interaction, and in general, the angular momentum diagrams with exchange interactions have topologically richer structure.

the expression shown in Fig. 9. The result, like the previous example, consist of Kronecker delta, phase factor and the same free part. However, unlike in the previous case, there is a $6 j$-symbol in the present example.

As the last example, consider the diagram in Fig. 2(a), which is a part of the $T_{2}^{(0)}$ equation. The corresponding angular momentum diagram is shown in Fig. 10. After angular integration, we get the expression shown in Fig. 11. The expression is similar in structure to the previous one, however, the free part shows the representa- $\delta\left(l_{1}, 0\right) \frac{\delta\left(l_{2}, l_{3}\right)}{\sqrt{\left[l_{2}\right]}}(-1)^{\left(-j_{b}+j_{q}+j_{c}+j_{r}\right)}\left\{\begin{array}{lll}k_{1} & j_{b} & j_{r} \\ l_{2} & j_{c} & j_{q}\end{array}\right\} \times j_{a}$

FIG. 9. Expression obtained after angular integration, using diagrammatic techniques, of the angular momentum diagram in Fig. 8.

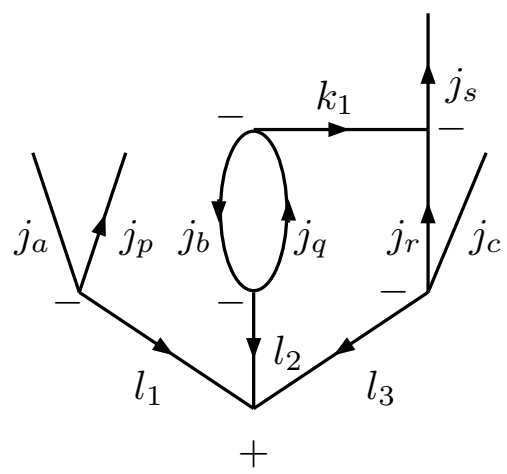

FIG. 10. Angular momentum diagram of the Goldstone diagram in Fig. 2(a) corresponding to the $T_{2}^{(0)}$ cluster amplitude.

tion adopted for the angular part of $T_{2}^{(0)}$. Following the same notations and angular momentum diagram evaluation rules, the angular integration of the Goldstone diagrams in the $T_{3}^{(0)}$ cluster equations are carried out.

TABLE I. The $\alpha_{0}$ and $\beta$ parameters of the even tempered GTO basis used in the present calculations.

\begin{tabular}{ccccccc}
\hline \hline Atom & \multicolumn{2}{c}{$s$} & \multicolumn{2}{c}{$p$} & \multicolumn{2}{c}{$d$} \\
& $\alpha_{0}$ & $\beta$ & $\alpha_{0}$ & $\beta$ & $\alpha_{0}$ & $\beta$ \\
\hline $\mathrm{Mg}$ & 0.02950 & 1.630 & 0.09750 & 1.815 & 0.00750 & 2.710 \\
$\mathrm{Ca}$ & 0.02050 & 1.970 & 0.05250 & 1.890 & 0.00690 & 2.695 \\
$\mathrm{Sr}$ & 0.01850 & 2.030 & 0.04750 & 2.070 & 0.00910 & 2.090 \\
$\mathrm{Ba}$ & 0.00925 & 2.110 & 0.00975 & 2.040 & 0.00995 & 2.010 \\
$\mathrm{Ra}$ & 0.00985 & 1.990 & 0.00925 & 1.980 & 0.00950 & 1.870 \\
\hline
\end{tabular}

\section{B. Basis set and nuclear density}

In the present work we use the Dirac-Hartree-Fock Hamiltonian and even-tempered Gaussian type orbitals (GTOs) [30]. The properties of the GTO's are described in our previous works $[12,17]$ and here, we highlight the main points. The large component of the Dirac spinorbitals are linear combinations of the Gaussian type functions

$$
g_{\kappa p}^{L}(r)=C_{\kappa i}^{L} r^{n_{\kappa}} e^{-\alpha_{p} r^{2}}
$$

where $p$ is the index of the Gaussian type function and $C_{\kappa i}^{L}$ is the normalization constant. The exponent $\alpha_{p}$ depends on two parameters $\alpha_{0}$ and $\beta$, these are related as 


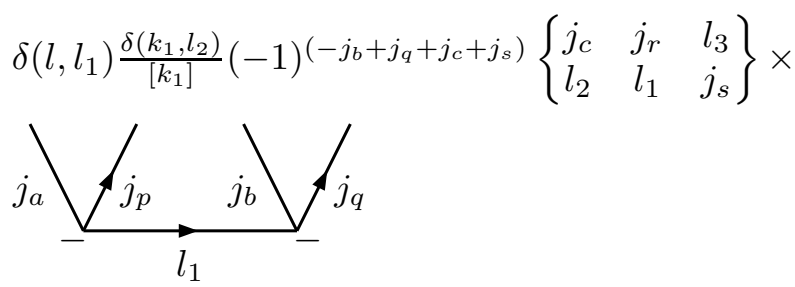

FIG. 11. Expression obtained after angular momentum integration, using diagrammatic techniques, of the diagram in Fig. 10

$\alpha_{p}=\alpha_{0} \beta^{p-1}$, where $p=0,1 \ldots m$ and $m$ is the number of the Gaussian type functions. The small components of the spin-orbitals are linear combination of $g_{\kappa p}^{S}(r)$, which are generated from $g_{\kappa p}^{L}(r)$ through the kinetic balance condition [29]. We compute the GTOs on a grid [38] with $V^{\mathrm{N}}$ potential and optimize the values of $\alpha_{0}$ and $\beta$ of each atom to match the spin-orbital energies and self consistent field (SCF) energy obtained from GRASP2K [39]. The latter solves the Dirac-Hartree-Fock equations numerically and for better convergence we use the HartreeFock orbitals [40] as the starting values of GRASP2K. The symmetry wise values of the optimized $\alpha_{0}$ and $\beta$ are listed in Table. I. Here we must emphasize that the proper optimization of $\alpha_{0}$ and $\beta$ is important as the quality of Dirac-Hartree-Fock orbitals depend on these parameters. The SCF energies with the optimized basis set

TABLE II. Comparison between GTO and GRASP2K SCF Energies

\begin{tabular}{lrr}
\hline Atom & \multicolumn{1}{c}{ GTO } & GRASP2K \\
\hline $\mathrm{Mg}$ & -199.9304 & -199.9351 \\
$\mathrm{Ca}$ & -679.7100 & -679.7102 \\
$\mathrm{Sr}$ & -3178.0789 & -3178.0801 \\
$\mathrm{Ba}$ & -8135.6412 & -8135.6475 \\
$\mathrm{Ra}$ & -25028.0819 & -25028.1072 \\
\hline
\end{tabular}

parameters are given in Table. II. The deviation of the GTO results is largest in the case of $\mathrm{Ra}$ and differs from the GRASP2K result by 0.0253 a.u. The orbital energies are, however, in excellent agreement. As example, the comparison of the orbital energies of $\mathrm{Ba}$ and $\mathrm{Ra}$ are listed in Table. III. The largest deviation is observe in the $1 s_{1 / 2}$ orbital energies of $\mathrm{Ba}$ and Ra. For the outer orbitals the difference in the orbital energies is below $10^{-4}$ a.u., which is evident from the table. A similar pattern is observed for $\mathrm{Mg}, \mathrm{Ca}$ and $\mathrm{Sr}$.

In addition to the basis parameter, the size of the orbital basis set is another important factor to obtain accurate results. In the present work, we chose the optimal orbital basis size after examining the convergence of $\alpha$. For example, in the case of $\mathrm{Ca}$ we start with a basis set size of 95 and increases it up to 165. The value of $\alpha$ with Dirac-Coulomb Hamiltonian is listed in Table. IV. It is evident from the table that, the optimal orbital basis

TABLE III. Orbital energies of $\mathrm{Ba}$ and $\mathrm{Ra}$ obtained from GRASP2K [39] and GTO in atomic units.

\begin{tabular}{|c|c|c|c|c|}
\hline \multirow[t]{2}{*}{ Orbital } & \multicolumn{2}{|c|}{$\mathrm{Ra}$} & \multicolumn{2}{|c|}{$\mathrm{Ba}$} \\
\hline & GTO & GRASP2K & GTO & GRASP2K \\
\hline $1 s_{1 / 2}$ & -3845.8119 & -3845.8206 & -1383.8341 & -1383.8358 \\
\hline $2 s_{1 / 2}$ & -712.6594 & -712.6607 & -222.5774 & -222.5777 \\
\hline $2 p_{1 / 2}$ & -685.0966 & -685.0966 & -209.0878 & -209.0881 \\
\hline $2 p_{3 / 2}$ & -571.9348 & -571.9345 & -195.0103 & -195.0103 \\
\hline $3 s_{1 / 2}$ & -179.6860 & -179.6863 & -48.6517 & -48.6517 \\
\hline $3 p_{1 / 2}$ & -167.2343 & -167.2343 & -42.9566 & -42.9566 \\
\hline $3 p_{3 / 2}$ & -141.1856 & -141.1855 & -40.1674 & -40.1673 \\
\hline $3 d_{3 / 2}$ & -121.3160 & -121.3158 & -30.2980 & -30.2979 \\
\hline $3 d_{5 / 2}$ & -115.9592 & -115.9590 & -29.7120 & -29.7119 \\
\hline $4 s_{1 / 2}$ & -45.6825 & -45.6827 & -10.2572 & -10.2572 \\
\hline $4 p_{1 / 2}$ & -40.1160 & -40.1161 & -8.0991 & -8.0991 \\
\hline $4 p_{3 / 2}$ & -33.3984 & -33.3984 & -7.5132 & -7.5132 \\
\hline $4 d_{3 / 2}$ & -24.4189 & -24.4188 & -3.9137 & -3.9135 \\
\hline $4 d_{5 / 2}$ & -23.1605 & -23.1604 & -3.8126 & -3.8126 \\
\hline $4 f_{5 / 2}$ & -11.3559 & -11.3560 & & \\
\hline $4 f_{7 / 2}$ & -11.0465 & -11.0465 & & \\
\hline $5 s_{1 / 2}$ & -10.0018 & -10.0020 & -1.6035 & -1.6035 \\
\hline $5 p_{1 / 2}$ & -7.8439 & -7.8440 & -0.9564 & -0.9564 \\
\hline $5 p_{3 / 2}$ & -6.3733 & -6.3734 & -0.8727 & -0.8727 \\
\hline $5 d_{3 / 2}$ & -3.1177 & -3.1177 & & \\
\hline $5 d_{5 / 2}$ & -2.9028 & -2.9028 & & \\
\hline $6 s_{1 / 2}$ & -1.6247 & -1.6247 & -0.1632 & -0.1632 \\
\hline $6 p_{1 / 2}$ & -0.9740 & -0.9740 & & \\
\hline $6 p_{3 / 2}$ & -0.7406 & -0.7406 & & \\
\hline $7 s_{1 / 2}$ & -0.1662 & -0.1662 & & \\
\hline
\end{tabular}

size at which $\alpha$ saturates to the level of $10^{-2}$ a.u. is 137 . As we increase beyond this, the change in $\alpha$ is $\leqslant 10^{-3}$ a.u. So, we use the orbital basis size 137 for further computations with the DCB Hamiltonian and to study the contributions from $T_{3}^{(0)}$.

TABLE IV. Convergence pattern of $\alpha(\mathrm{Ca})$ as a function of the basis set size.

\begin{tabular}{lcc}
\hline No. of orbitals & Basis size & $\alpha$ \\
\hline 95 & $(13 s, 11 p, 9 d, 7 f, 7 g, 7 h)$ & 163.74 \\
113 & $(15 s, 13 p, 11 d, 9 f, 9 g, 7 h)$ & 163.55 \\
127 & $(17 s, 15 p, 11 d, 11 f, 9 g, 9 h)$ & 163.53 \\
137 & $(19 s, 15 p, 13 d, 11 f, 11 g, 9 h)$ & 163.52 \\
147 & $(21 s, 17 p, 17 d, 13 f, 11 g, 11 h)$ & 163.52 \\
165 & $(23 s, 19 p, 15 d, 13 f, 13 g, 11 h)$ & 163.52 \\
\hline
\end{tabular}

In the present work we use the finite size Fermi density distribution of the nucleus

$$
\rho_{\mathrm{nuc}}(r)=\frac{\rho_{0}}{1+e^{(r-c) / a}}
$$

where, $a=t 4 \ln (3)$. The parameter $c$ is the half charge radius so that $\rho_{\text {nuc }}(c)=\rho_{0} / 2$ and $t$ is the skin thickness. In one of our earlier works [14] we had examined the vacuum polarization corrections to the orbital energies and 
atomic properties. In the present work, we neglect the vacuum polarization effects as we find the corrections in the properties of neutral alkaline atoms are very small. The PRCC equations are solved iteratively using Jacobi method, we have chosen this method as it is easily parallelizable. The method, however, is slow to converge. So, we use direct inversion in the iterated subspace (DIIS) [41] to accelerate the convergence.

\section{Convergence criteria}

The computation of $\alpha$, as described earlier, involves several steps: the generation of orbital basis set; computation of the RCC cluster amplitudes $\left(T^{(0)}\right)$; and computation of PRCC cluster amplitudes $\left(\mathbf{T}^{(1)}\right)$. Each of these are iterative in nature and involves the choice of a convergence parameter $\epsilon$. In the SCF computations to generate the orbital basis set, $\epsilon$ is the maximum change in the orbital between two consecutive iterations, and in the computations of $T^{(0)}$ and $\mathbf{T}^{(1)}$, it is the average change in the cluster amplitudes between consecutive iterations. In all of these we set choose $\epsilon \leqslant 10^{-6}$ and it is computationally manageable with single and double excitation approximation in the coupled-cluster computation. However, with the the inclusion of the triple excitations, we choose $\epsilon \leqslant 10^{-5}$. This choice is compelled by the computational requirements and the effect on the value of $\alpha$ is below $10^{-4}$ a.u. To elaborate further, when $T_{3}^{(0)}$ is included, the number of cluster amplitudes increases by several order of magnitudes and each iteration requires thousands of compute hours. For example, in $\mathrm{Ca}$, consider the orbital basis with 137 orbitals, the optimal size to obtain converged value of $\alpha$. With this basis set, number of the $T_{1}^{(0)}$ and $T_{2}^{(0)}$ cluster amplitudes are $\approx 10^{2}$ and $\approx 10^{5}$, respectively. On the other hand, the number of $T_{3}^{(0)}$ cluster amplitudes is $\approx 10^{10}$. So, it involves massive computational operations and in addition, due to the large number we observe slower convergence. We find that even with the reduction in the number of $T_{3}^{(0)}$ to $\approx 10^{8}$, based on energy considerations, solving the cluster equations takes weeks on multi-node cluster computers. So, decreasing $\epsilon$ by an order magnitude to $10^{-5}$ reduces a few iterations, and we can solve the cluster equations in about three weeks using 64 processors on a cluster computer.

\section{Computational issues related to $T_{3}^{(0)}$}

As discussed in the previous section, to compute the $T_{3}^{(0)}$ cluster amplitudes within reasonable time, we chose dominant $T_{3}^{(0)}$ amplitudes based on the orbital energies. For this consider the perturbative approximation of the
$T_{3}^{(0)}$ cluster amplitude

$$
t_{a b c}^{p q r} \approx \frac{1}{\Delta E}\left(\sum_{s} t_{a b}^{p s} g_{s c}^{q r}+\sum_{d} t_{a d}^{p q} g_{b c}^{d r}\right)
$$

where, as defined earlier $t_{a b}^{p q}$ is the $T_{2}^{(0)}$ cluster amplitude, $g_{i j}^{k l}=\left\langle k l\left|1 / r_{12}+g^{B}\left(r_{12}\right)\right| i j\right\rangle$, and the energy denominator is

$$
\Delta E=\left(\varepsilon_{p}+\varepsilon_{q}+\varepsilon_{r}-\varepsilon_{a}-\varepsilon_{b}-\varepsilon_{c}\right) .
$$

In the above expression, $\varepsilon_{i}$ is the single particle energy of the $i$ th orbital. Since, $t_{a b c}^{p q r} \propto 1 / \Delta E$, the dominant contributions arise from small $\Delta E$ and for the present work we select the orbitals such that $\Delta E \leqslant 100$ a.u. Here, we have set the selection criterion based on $\Delta E$ as the numerators $t_{a b}^{p s} g_{s c}^{q r}$ and $t_{a d}^{p q} g_{b c}^{d r}$ are, in general, smaller than one when $\Delta E>10$.

There is an important consideration associated with the $T_{3}^{(0)}$ cluster amplitudes during the computation. This is related to the maximum occupancy of the $s_{1 / 2}$ and $p_{1 / 2}$ orbitals, which is two, to avoid violation of Pauli's exclusion principle. During computations we ensure that not all the $a, b$ and $c$ in $t_{a b c}^{p q r}$ are associated with the same $s_{1 / 2}$ or $p_{1 / 2}$ sub-shells. Similar consideration must be made for the $p, q$ and $r$. Implementing this explicitly as a selection rule, however, leads to larger computational operations as $t_{a b c}^{p q r}$ contributes to single, double and triple excitation cluster amplitudes. A more efficient and faster scheme is to allow an un-constrained computation of the terms in the cluster equations, including the terms with triple occupancy of $s_{1 / 2}$ and $p_{1 / 2}$ sub-shells. We then, subtract these terms just before computing the new values of the cluster amplitudes. This method of selected subtraction speeds up computation as it avoids global implementation of a selection rule requiring multiple conditional statements.

\section{RESULTS AND DISCUSSIONS}

In the present work, we use the expression of $\alpha$ in Eq. (20), which consider terms up to second order in cluster operators. This is a natural choice as the contributions from the higher order terms are very small $\approx 10^{-4}$ a.u. It is important to mention that unlike the closed-shell noble gas atoms and one-valence alkali atoms, the alkaline earth metal atoms are two-valence systems and have more complex structure. Further more, the uncertainties in the experimental results are also large. So, it is important to identify and quantify the theoretical uncertainties in the computations. In table $\mathrm{V}$ we list the $\alpha$ of alkaline-Earth metal atoms $\mathrm{Mg}, \mathrm{Ca}, \mathrm{Sr}, \mathrm{Ba}$ and $\mathrm{Ra}$ computed using the PRCC expression of $\alpha$. In the second column of the table we give our results with two different sets of calculations : one is with the Dirac-Coulomb (DC) Hamiltonian, and the other is with the Dirac-CoulombBreit (DCB) Hamiltonian. For a systematic comparison 
TABLE V. Static dipole polarizability $\alpha$ of alkaline-Earthmetal atoms and the values are in atomic units. The results from this work identified with $\mathrm{C}$ and $\mathrm{B}$ within parenthesis indicate the use of Dirac-Coulomb and Dirac-Coulomb-Breit Hamiltonian, respectively.

\begin{tabular}{|c|c|c|c|c|}
\hline Atom & This work & Method & Previous Works & Method \\
\hline \multirow[t]{5}{*}{$\overline{\mathrm{Mg}}$} & 71.64 & $\overline{\mathrm{PRCC}(\mathrm{C})}$ & $71.35^{\mathrm{a}}$ & $\overline{\mathrm{CICP}}$ \\
\hline & 70.76 & $\operatorname{PRCC}(\mathrm{B})$ & $70.90^{\mathrm{b}}$ & \\
\hline & & & $72.54^{\mathrm{c}}$ & RCCSD \\
\hline & & & $71.33^{\mathrm{d}}$ & $\mathrm{RCI}+\mathrm{MBPT}$ \\
\hline & & & $71.5(3.1)^{\mathrm{e}}$ & Expt. \\
\hline \multirow[t]{5}{*}{$\mathrm{Ca}$} & 163.52 & $\operatorname{PRCC}(\mathrm{C})$ & $158.00^{f}$ & RCCSDT \\
\hline & 160.77 & $\operatorname{PRCC}(\mathrm{B})$ & $152.0^{\mathrm{g}}$ & RCCSDT \\
\hline & & & $157.03^{\mathrm{c}}$ & RCCSD \\
\hline & & & $159.0^{\mathrm{d}}$ & $\mathrm{RCI}+\mathrm{MBPT}$ \\
\hline & & & $169(17)^{\mathrm{h}}$ & Expt. \\
\hline \multirow[t]{5}{*}{$\mathrm{Sr}$} & 192.40 & $\operatorname{PRCC}(\mathrm{C})$ & $198.85^{f}$ & RCCSDT \\
\hline & 190.82 & $\operatorname{PRCC}(\mathrm{B})$ & $190^{\mathrm{g}}$ & RCCSDT \\
\hline & & & $186.98^{\mathrm{c}}$ & RCCSD \\
\hline & & & $202.0^{\mathrm{d}}$ & $\mathrm{RCI}+\mathrm{MBPT}$ \\
\hline & & & $186(15)^{\mathrm{i}}$ & Expt. \\
\hline \multirow[t]{5}{*}{$\mathrm{Ba}$} & 278.24 & $\operatorname{PRCC}(\mathrm{C})$ & $273.9^{f}$ & RCCSDT \\
\hline & 274.68 & $\operatorname{PRCC}(\mathrm{B})$ & $275.5^{\mathrm{j}}$ & RCCSDT \\
\hline & & & $268.19(7.28)^{\mathrm{k}}$ & RCCSD \\
\hline & & & $272.1^{\mathrm{d}}$ & $\mathrm{RCI}+\mathrm{MBPT}$ \\
\hline & & & $268(22)^{\mathrm{i}}$ & Expt. \\
\hline \multirow[t]{2}{*}{$\mathrm{Ra}$} & 243.52 & $\operatorname{PRCC}(\mathrm{C})$ & $248.56^{\mathrm{f}}$ & RCCSDT \\
\hline & 242.42 & $\operatorname{PRCC}(\mathrm{B})$ & & \\
\hline
\end{tabular}

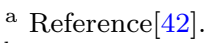

b Reference[43].

${ }^{c}$ Reference[44].

d Reference[45].

e Reference[46].

${ }^{\mathrm{f}}$ Reference[47].

g Reference[48].

${ }^{\mathrm{h}}$ Reference[49].

${ }^{i}$ Reference[50].

j Reference[51].

${ }^{\mathrm{k}}$ Reference[15].

we also list the previous theoretical and experimental results.

For a more detailed study, we examine the contributions from each of the terms in the Eq. (20) and these are listed in Table. VI. In all the cases, the leading order (LO) term is $\mathbf{T}_{1}^{(1) \dagger} \mathbf{D}+$ h.c. This is natural as these terms subsume the contributions from the Dirac-Fock and RPA effects. Further more, the contribution from the LO term exceeds the final value of $\alpha$ and a similar trend was observed in our earlier work on noble gas atoms [11, 12] as well as the alkali-metal [13] and alkaline-earth-metal [14] ions. To examine the contribution from $\mathbf{T}_{1}^{(1) \dagger} \mathbf{D}$ in finer detail, we separate out and list the contribution from each of the occupied orbitals to this term in the Table. VII. In all the cases, the valence orbital surpasses contributions from the other occupied orbitals by orders magnitude. More importantly, in all the cases, the con-
TABLE VI. Contribution to $\alpha$ from different terms and their hermitian conjugates in the PRCC theory.

\begin{tabular}{lrrrrr}
\hline Terms + h.c. & $\mathrm{Mg}$ & $\mathrm{Ca}$ & $\mathrm{Sr}$ & $\mathrm{Ba}$ & $\mathrm{Ra}$ \\
\hline $\mathbf{T}_{1}^{(1) \dagger} \mathbf{D}$ & 75.790 & 185.852 & 224.452 & 342.336 & 311.590 \\
$\mathbf{T}_{1}{ }^{(1) \dagger} \mathbf{D} T_{2}^{(0)}$ & -2.400 & -7.254 & -9.130 & -18.674 & -16.462 \\
$\mathbf{T}_{2}{ }^{(1) \dagger} \mathbf{D} T_{2}^{(0)}$ & 2.706 & 10.004 & 12.926 & 30.348 & 23.506 \\
$\mathbf{T}_{1}{ }^{(1) \dagger} \mathbf{D} T_{1}^{(0)}$ & -2.056 & -9.926 & -12.522 & -29.008 & -27.200 \\
$\mathbf{T}_{2}^{(1) \dagger} \mathbf{D} T_{1}^{(0)}$ & 0.184 & 0.906 & 1.236 & 3.238 & 3.104 \\
Normalization & 1.049 & 1.117 & 1.137 & 1.195 & 1.215 \\
Total & 70.757 & 160.771 & 190.820 & 274.678 & 242.418 \\
\hline
\end{tabular}

tributions from the inner occupied orbitals are opposite to that of the valence orbitals.

On examining the trend in the next to leading order (NLO) term, there is a significant departure from the general trend observed in our previous works on noble gas atoms [11, 12], alkali-metal ions [13] and alkalineearth-metal ions [14]. In these systems the NLO term is $\mathbf{T}_{1}{ }^{(1) \dagger} \mathbf{D} T_{2}^{(0)}$ and contribution is opposite in phase to the LO term. However, in neutral alkaline atoms, as evident from Table. V, the NLO term is $\mathbf{T}_{2}{ }^{(1) \dagger} \mathbf{D} T_{2}^{(0)}$ and the contribution has the same phase as the LO term. Another important point is, the NLO and the other important terms, $\mathbf{T}_{1}{ }^{(1) \dagger} \mathbf{D} T_{1}^{(0)}$ and $\mathbf{T}_{1}{ }^{(1) \dagger} \mathbf{D} T_{2}^{(0)}$ have much larger contributions compared to the systems studied earlier. For better presentation of the detailed analysis of the results, we separate the discussion into three groups: $\mathrm{Mg}$ and $\mathrm{Ca}$, $\mathrm{Sr}$ and $\mathrm{Ba}$, and $\mathrm{Ra}$.

TABLE VII. Four leading contributions to $\left\{\mathbf{T}_{1}^{(1) \dagger} \mathbf{D}\right\}$ to $\alpha$ in terms of the core spin-orbitals.

\begin{tabular}{ccc}
\hline $\mathrm{Mg}$ & $\mathrm{Ca}$ & $\mathrm{Sr}$ \\
\hline $38.308\left(3 s_{1 / 2}\right)$ & $95.257\left(4 s_{1 / 2}\right)$ & $114.342\left(5 s_{1 / 2}\right)$ \\
$-0.032\left(2 p_{3 / 2}\right)$ & $-0.529\left(3 p_{3 / 2}\right)$ & $-0.715\left(4 p_{3 / 2}\right)$ \\
$-0.016\left(2 p_{1 / 2}\right)$ & $-0.266\left(3 p_{1 / 2}\right)$ & $-0.336\left(4 p_{1 / 2}\right)$ \\
$-0.007\left(2 s_{1 / 2}\right)$ & $-0.012\left(3 s_{1 / 2}\right)$ & $-0.012\left(3 d_{5 / 2}\right)$ \\
\hline $\mathrm{Ba}$ & $\mathrm{Ra}$ & \\
\hline $175.500\left(6 s_{1 / 2}\right)$ & $157.974\left(7 s_{1 / 2}\right)$ & \\
$-1.400\left(5 p_{3 / 2}\right)$ & $-0.760\left(6 p_{3 / 2}\right)$ & \\
$-0.567\left(5 p_{1 / 2}\right)$ & $-0.202\left(6 p_{1 / 2}\right)$ & \\
$-0.072\left(4 d_{5 / 2}\right)$ & $-0.101\left(5 d_{5 / 2}\right)$ & \\
\hline
\end{tabular}

\section{A. $\mathrm{Mg}$ and $\mathrm{Ca}$}

The results for $\alpha$ of Mg computed with the DC Hamiltonian is in excellent agreement with the experimental value. With $H^{\mathrm{DCB}}$, the value of $\alpha$ is lowered by -0.88 a.u., which is not negligible. This is in contrast to the case of noble gas atoms, for which we observed an increase in $\alpha$ when Breit interaction is included [12]. This 
is due to the different orbital angular momentum of the valence shell, which has the leading order contribution to $\alpha$. More precisely, the $3 s$ orbital in $\mathrm{Mg}$ is radially contracted when Breit interaction is included, where as in the noble gas atoms the valence orbital $\left(n p_{3 / 2}\right)$ is radially dilated with Breit interaction. Our results for $\mathrm{Mg}$ is in good agreement with the previous theoretical values, including the results from a relativistic coupled-cluster computations [44]. In particular, the DC result of $\mathrm{Mg}$ is in very good agreement with the recommended value of a previous theoretical work [45] as well as the results from configuration interaction with semi empirical core potential model [42], however, the DCB result is $0.8 \%$ lower than the recommended value. Among the various terms in the PRCC expression of $\alpha$, as described earlier, the LO term is $\mathbf{T}_{1}{ }^{(1) \dagger} \mathbf{D}$. On examining further, based on the values listed in Table. VII, almost the total value of $\alpha$ arises from the core orbital $3 s$. The contribution from the next core shell $2 p_{3 / 2}$, in terms of energy, is $\approx 0.08 \%$, which is negligible. At a finer level, the five dominant $\tau_{a}^{p}$ in $\mathbf{T}_{1}{ }^{(1) \dagger} \mathbf{D}$ are listed in Table. VIII. According to the table, the dominant contributions arise from the $\tau_{3 s_{1 / 2}}^{3 p_{3 / 2}}$ and $\tau_{3 s_{1 / 2}}^{3 p_{1 / 2}}$ cluster amplitudes.

TABLE VIII. Orbitals contribution from $\mathbf{T}_{1}{ }^{(1) \dagger} \mathbf{D}$ to $\alpha$ of $\mathrm{Mg}$ and $\mathrm{Ca}$

\begin{tabular}{rrr}
\hline $\mathrm{Mg}$ & $\mathrm{Ca}$ \\
\hline $25.475\left(3 s_{1 / 2}, 3 p_{3 / 2}\right)$ & $62.072\left(4 s_{1 / 2}, 4 p_{3 / 2}\right)$ \\
$12.780\left(3 s_{1 / 2}, 3 p_{1 / 2}\right)$ & $33.159\left(4 s_{1 / 2}, 4 p_{1 / 2}\right)$ \\
$0.032\left(3 s_{1 / 2}, 4 p_{3 / 2}\right)$ & $-0.229\left(3 p_{3 / 2}, 6 d_{3 / 2}\right)$ \\
$0.016\left(3 s_{1 / 2}, 4 p_{1 / 2}\right)$ & $-0.138\left(3 p_{3 / 2}, 5 d_{3 / 2}\right)$ \\
\hline $\mathrm{Sr}$ & $\mathrm{Ba}$ \\
\hline $73.370\left(5 s_{1 / 2}, 5 p_{3 / 2}\right)$ & $76.628\left(6 s_{1 / 2}, 6 p_{3 / 2}\right)$ \\
$40.975\left(5 s_{1 / 2}, 5 p_{1 / 2}\right)$ & $45.697\left(6 s_{1 / 2}, 6 p_{1 / 2}\right)$ \\
$-0.236\left(4 p_{3 / 2}, 6 d_{5 / 2}\right)$ & $32.977\left(6 s_{1 / 2}, 7 p_{3 / 2}\right)$ \\
$-0.176\left(4 p_{3 / 2}, 7 d_{5 / 2}\right)$ & $16.538\left(6 s_{1 / 2}, 7 p_{1 / 2}\right)$ \\
\hline \multicolumn{2}{c}{$\mathrm{Ra}$} \\
\hline $48.128\left(7 s_{1 / 2}, 7 p_{3 / 2}\right)$ & \\
$45.844\left(7 s_{1 / 2}, 7 p_{1 / 2}\right)$ & \\
$34.735\left(7 s_{1 / 2}, 8 p_{3 / 2}\right)$ & \\
$22.021\left(7 s_{1 / 2}, 8 p_{1 / 2}\right)$ & \\
\hline
\end{tabular}

For $\mathrm{Ca}$, there are three previous results based on $\mathrm{RCC}$ theory [44, 47, 48], however, there is a variation among the results. Compared to these previous results, our result of $\alpha$ with the DC Hamiltonian is on the higher side, but with the inclusion of Breit interaction our result is in good agreement with the values given in ref. [47] and [44]. In particular, our result is $1.7 \%$ higher than the RCCSDT result obtained with finite field method [47] with scalar relativistic Douglas-Kroll Hamiltonian, which is a different approach compared to our method. Further more, our result is $1.1 \%$ higher that result from calculations with hybrid RCI+MBPT method [45].

When compared to $\mathrm{Mg}$, in Ca we notice a large increase in the value of $\alpha$. This may be understood in terms of the difference in the properties of the valence orbitals generated with the GTO or numerically using GRASP2K. In $\mathrm{Mg}$, the valence orbital $3 s$ has an energy of -0.255 hartree and average radial extent $\langle r\rangle=3.252 \mathrm{a}_{0}$. For $\mathrm{Ca}$, the valence orbital is $4 s$, and the energy and $\langle r\rangle$ are -0.198 hartree and $4.191 \mathrm{a}_{0}$, respectively. Thus, the valence orbital of $\mathrm{Ca}$ is $\approx 30 \%$ larger in size, and as $\alpha \propto r^{2}$, we can expect larger $\alpha$. This is reflected in the dominant $\mathbf{T}_{1}^{(1)}$ cluster amplitude which contributes to the leading order term in $\alpha$, namely $\mathbf{T}_{1}{ }^{(1) \dagger} \mathbf{D}$ and it's complex conjugate. In Ca, the contribution from $\tau_{4 s_{1 / 2}}^{4 p_{3 / 2}}$ to $\alpha, \mathbf{T}_{1}^{(1) \dagger} \mathbf{D}$ is 62.072. This is $\approx 2.5$ times larger than the dominant contribution in $\mathrm{Mg}$ arising from the $\tau_{3 s_{1 / 2}}^{3 p_{3 / 2}}$ cluster amplitude. A similar trend is observe in the second most dominant cluster amplitude.

\section{B. Sr and $\mathrm{Ba}$}

In the case of $\mathrm{Sr}$ too, like in $\mathrm{Ca}$, there is a variation in the previous theoretical results obtained from RCC theory $[44,47,48]$. However, unlike in $\mathrm{Ca}$, our result with DC Hamiltonian lies between the previous RCC results, and with Breit interaction our result is almost an exact match with the RCC result [48] using Cowan-Griffin approximation [52]. This is as expected since the relativistic corrections, though important, are not very large for neutral atoms like $\mathrm{Sr}$ with nuclear charge $Z=38$ as $Z \alpha<1$ (here, $\alpha$ is the fine structure constant). On a closer inspection, the Breit interaction contribution to $\alpha$ is -1.58 a.u., which is $\approx 0.8 \%$ of the total value and this is marginally lower than in Ca. This could be due to the screening effect from the electrons in the diffused $3 d$ shell.

The remaining two atoms in the group, $\mathrm{Ba}$ and $\mathrm{Ra}$ with nuclear charges 56 and 88, respectively, are good candidate atoms to examine the relativistic implementation of coupled-cluster theories in detail. In particular, the PRCC theory we have developed for properties calculations with an additional perturbation. For Ba, three of the previous results $[15,47,51]$ are based on RCC theory, and our results are consistent the previous results in ref. [47, 51]. Referring to the third RCC work [15], accounting for the theoretical uncertainty reported, we may consider our results consistent with the values reported in that work. However, a more detailed comparison is non-trivial as the normalization factor reported in ref. [15] is less than unity. Perhaps, this is on account of the scheme adopted to implement the relativistic coupledcluster theory in their work. On inclusion of Breit interaction, using DCB Hamiltonian, the value of $\alpha$ is reduced by -3.56 a.u., which is $\approx 1.3 \%$ of the value obtained with DC Hamiltonian. Our result with DCB Hamiltonian is in very good agreement with the previous theoretical result obtained from RCCSDT [47], more precisely, our result is $0.3 \%$ lower than the value reported in ref. [47]. On the other hand, our result is $0.9 \%$ higher than the result 
from the hybrid RCI+MBPT [45].

The relative change in $\alpha$ as we compare the value of $\mathrm{Ba}$ to $\mathrm{Sr}$ is not very remarkable. However, on closer examination, there is a pronounced change in the identity of the dominant $\tau_{a}^{p}$ contributing to the leading PRCC term $\mathbf{T}_{1}{ }^{(1) \dagger} \mathbf{D}$. Like in the other atoms discussed so far, as evident from Table. VIII, the two leading contributions arise from the cluster amplitudes with the outermost $s$ and $p$ orbitals. More precisely, these are the cluster amplitudes $\tau_{6 s_{1 / 2}}^{6 p_{3 / 2}}$ and $\tau_{6 s_{1 / 2}}^{6 p_{1 / 2}}$. The contributions from the next two dominant cluster amplitudes $\tau_{6 s_{1 / 2}}^{7 p_{3 / 2}}$ and $\tau_{6 s_{1 / 2}}^{7 p_{1 / 2}}$ are $\approx 43 \%$ and $\approx 22 \%$ of the most dominant $\left(\tau_{6 s_{1 / 2}}^{6 p_{3 / 2}}\right)$ contribution, respectively. This is very different from the trend observed in $\mathrm{Mg}, \mathrm{Ca}$ and $\mathrm{Sr}$, where the third dominant contribution is below $1 \%$ of the most dominant contribution. Further more, in the lighter atoms $\mathrm{Ca}$ and $\mathrm{Sr}$, the third dominant contribution arises from the cluster amplitude of the form $\tau_{3 p_{3 / 2}}^{5 d_{3 / 2}}$ and $\tau_{4 p_{3 / 2}}^{6 d_{5 / 2}}$, respectively. This could be on account of the relativistic contraction of the $s$ and $p$ orbitals. These results indicate that the choice of the basis set is very important to obtain reliable results in Ba.

\section{Ra}

We consider the case of $\mathrm{Ra}$ as the most important among the alkaline-earth metal atoms for the present study, and it is also the most challenging. The primary reasons are: importance of the relativistic effects; and absence of experimental data. Considering that $Z$ is 88 and $Z \alpha \approx 0.64$ (where $\alpha$ is the fine structure constant), it is absolutely essential to employ a relativistic description. For $\mathrm{Ra}$, there is one previous theoretical result based on RCCSDT using scalar relativistic Douglas-Kroll operator $\alpha$ [47]. As evident from Table. V, our result of $\alpha$ with DC Hamiltonian is $\approx 2 \%$ lower than the result in ref. [47]. On including the Breit interaction the difference increases to $2.5 \%$. An important observation is, compared to the case of $\mathrm{Ba}$ the value of $\alpha$ for $\mathrm{Ra}$ is $\approx 11.7 \%$ lower. A similar trend is observed in the results reported in ref. [47], in their case the $\alpha$ of $\mathrm{Ra}$ is $\approx 9.3 \%$ lower than Ba. This reduction is on account of the radial contraction of the $7 \mathrm{~s}$ orbital, the valence orbital of $\mathrm{Ra}$, due to the relativistic effects. This is evident from the $\langle r\rangle$ of the orbitals generated either with GTO or numerically using GRASP2K. In $\mathrm{Ra}$, the $\langle r\rangle$ of the valence orbital $7 s$ is $5.04 a_{0}$, this is lower than the value of $5.06 a_{0}$ for the valence orbital $6 s$ in $\mathrm{Ba}$.

To identify and as well as quantify the relative change from the $\alpha$ of $\mathrm{Ba}$, let us examine the dominant $\tau_{a}^{p}$ which contribute to the leading order term in PRCC $\mathbf{T}_{1}^{(1) \dagger} \mathbf{D}$. From the values listed in Table VIII, in Ra the most dominant contribution of 48.128 a.u. arises from the $\tau_{7 s_{1 / 2}}^{7 p_{3 / 2}}$ cluster amplitude. However, compared to the most dominant contribution of 76.628 a.u. in $\mathrm{Ba}$, arising from the $\tau_{6 s_{1 / 2}}^{6 p_{3 / 2}}$ cluster amplitude, it is $\approx 37.2 \%$ smaller. For the next three dominant $\tau_{a}^{p}$ cluster amplitudes, the contributions in $\mathrm{Ra}$ are on par with those of $\mathrm{Ba}$ or slightly. So, most of the changes or reduction in $\alpha$ can be attributed to the lower contribution from the dominant cluster amplitude in the LO term $\mathbf{T}_{1}^{(1) \dagger} \mathbf{D}$. Coming to the contribution from the Breit interaction, after it's inclusion the value of $\alpha$ is 1.10 a.u. less than the result with DC Hamiltonian.

\section{D. $T_{3}^{(0)}$ Contribution to $\alpha$}

Here we examine the contribution from $T_{3}^{(0)}$ to $\alpha$. For this we compute the most dominant contribution from $T_{3}^{(0)}$. In general, as we have shown earlier, the leading order contribution arises from the terms involving $T_{1}^{1}$ cluster operators. However, with $T_{3}^{(0)}$ only the terms with structural radiation diagrams can have non-zero contribution involving $T_{1}^{(1)}$. In general, the contributions from the structural radiation diagrams are negligible and not included in the present work. So, the dominant contribution from $T_{3}^{(0)}$ arises from the term $T_{3}^{(0) \dagger} \mathbf{D} \mathbf{T}_{2}^{(1)}$. The diagrams of $\alpha$ arising from this term are shown in Fig. 5. The computation with $T_{3}^{(0)}$ is restricted to the cluster amplitudes involving the outer core orbitals and low-lying virtual orbitals. This, as mentioned earlier, is due to the limitations of the computational resources. Even then, it takes close to a month to solve the $T_{3}^{(0)}$ on cluster computers for lighter atoms. So, in this work we report the results of $\alpha$ for only $\mathrm{Mg}$ and $\mathrm{Ca}$ with $T_{3}^{(0)}$. The contribution is small and it is 0.0016 a.u. and 0.0317 a.u for $\mathrm{Mg}$ and Ca, respectively. This may be on account of considering only the triple excitations of RCC amplitudes. On the other hand the contribution from $\mathbf{T}_{3}^{(1)}$ may not be small. We are in the process of developing a theory to incorporate $\mathbf{T}_{3}^{(1)}$, but computational implementation is non-trivial as the representation now involves four multipole operators.

\section{E. Uncertainty estimates}

To estimate the uncertainties associated with the present results, we have identified some important sources. These are associated with the various approximations at different levels of the computations. The first source of uncertainty in our computations is associated the truncation of the orbital basis set. To reduce this uncertainty we do a series of computations and as we discussed earlier, identify the optimal basis set size. So it is safe to neglect this uncertainty. The second source of uncertainty is, as we consider upto $T_{3}^{(0)}$ in the RCC theory, the contribution from the $T_{4}^{(0)}$ and other higher order excitations is a source of uncertainty. We have shown the contribution from the triple excitations is $\approx 10^{-2}$ for $\mathrm{Ca}$ 
and we expect a similar pattern for other heavy atoms, $\mathrm{Sr}, \mathrm{Ba}$ and $\mathrm{Ra}$. So the uncertainty associated with the $T_{4}^{(0)}$ and other higher order excitations can be ignored. The third source of uncertainty arises from the contribution of $\mathbf{T}_{3}^{(1)}$ and higher order excitation cluster operators in PRCC theory. Considering the results from the finite field calculations in ref. [47], the average contribution from $T_{3}^{(0)}$ is $\approx 2.8 \%$. So, in the present work we may take this as the upper bound on the uncertainty associated with the truncation of cluster operators in PRCC. The fourth source of uncertainty is associated with the truncation of the expression of $\alpha$ in PRCC theory to second order in cluster amplitudes. In one of our earlier studies [53] we observed the contribution from third order terms is negligibly small. The last, fifth, source of uncertainty is quantum electrodynamical(QED) corrections. However in one of our earlier work [14] we have shown the contribution from the vacuum polarization correction to the $\alpha$ is very small and it is less than $0.1 \%$.

For high- $Z$ atoms like Ra, there are two other possible sources of theoretical uncertainties. These are: frequency dependent part of the Breit interaction; and deviation from no-virtual-pair approximation in the atomic Hamiltonian. To estimate the uncertainty from the first, we do a series of calculations using GRASP2K [39], which has the option of including and scaling the frequency dependent part of Breit interaction. Based on the calculations, for $\mathrm{Ra}$ we estimate the upper bound on the contribution from frequency dependent part of the Breit interaction to $\alpha$ as $0.13 \%$. Similarly, for $\mathrm{Ba}$ it is $0.09 \%$ and can be neglected for the other lighter atoms. To estimate the second possible source-deviation from the no-virtual-pair approximation-we consider the leading order diagram of $\alpha$ and find that the contribution from diagrams with virtual pairs is less than $10^{-4}$ a.u. for Ra. This can be neglected and perhaps, the contribution may be higher for the $\alpha$ of highly charged ions. Combining all the sources, we estimate the uncertainty of our results to be below
$2.9 \%$.

\section{CONCLUSION}

We have studied the static dipole polarizability of alkaline Earth metal atoms. For this we use the PRCC theory, we have developed, to incorporate the external perturbation in an atomic or ionic system. We then, examine the contributions from $T_{3}^{(0)}$ based on a symmetric representation we have developed and defined the corresponding angular momentum representation suitable for atomic calculations. The RCCSDT theory reported in this work can be applied to study other properties like hyperfine structure constants, and extend to one- and two-valence [54] atoms and ions as well. These are in progress and we shall report in our future works.

Our results of the $\alpha$ of alkaline Earth metal atoms are in very good agreement with the previous theoretical values and it is also within the uncertainty limit of the experimental data. This is perhaps not surprising as the PRCC theory has produced excellent results for closed shell noble gas atoms as well as singly ionized alkali metal ions and doubly charged alkaline Earth metal ions [11-14]. We examine the contribution from the Breit interaction for each of the alkaline Earth metal atoms and find that it is not negligible. For these atoms, the Breit interaction decreases the value of $\alpha$, this is opposite to the trend observed in noble gas atoms.

\section{ACKNOWLEDGMENTS}

We thank S. Gautam, Arko Roy and Kuldeep Suthar for useful discussions. The results presented in the paper are based on the computations using the 3TFLOP HPC Cluster at Physical Research Laboratory, Ahmedabad.
[1] K. Bonin and V. Kresin, Electric-Dipole Polarizabilities of Atoms, Molecules and Clusters (World Scientific Publ., Singapore, 1997).

[2] V. A. Dzuba, V. V. Flambaum, and J. S. M. Ginges, Phys. Rev. A 61, 062509 (2000).

[3] J. Ginges and V. Flambaum, Phys. Rep. 397, 63 (2004).

[4] A. D. Ludlow, T. Zelevinsky, G. K. Campbell, S. Blatt, M. M. Boyd, M. H. G. de Miranda, J. W. Martin, M. J.and Thomsen, S. M. Foreman, J. Ye, T. M. Fortier, J. E. Stalnaker, S. A. Diddams, Y. Le Coq, Z. W. Barber, N. Poli, N. D. Lemke, K. M. Beck, and C. W. Oates, Science 319, 1805 (2008).

[5] G. Wilpers, C. W. Oates, S. A. Diddams, A. Bartels, T. M. Fortier, W. H. Oskay, J. C. Bergquist, S. R. Jefferts, T. P. Heavner, T. E. Parker, and L. Hollberg, Metrologia 44, 146151 (2007).

[6] Y. N. M. de Escobar, P. G. Mickelson, M. Yan, B. J.
DeSalvo, S. B. Nagel, and T. C. Killian, Phys. Rev. Lett. 103, 200402 (2009).

[7] S. Stellmer, M. K. Tey, B. Huang, R. Grimm, and F. Schreck, Phys. Rev. Lett. 103, 200401 (2009).

[8] S. Kraft, F. Vogt, O. Appel, F. Riehle, and U. Sterr, Phys. Rev. Lett. 103, 130401 (2009).

[9] J. Mitroy, M. S. Safronova, and C. W. Clark, J. Phys. B 43, 202001 (2010).

[10] P. Schwerdtfeger, "Atomic static dipole polarizabilities," in Computational Aspects of Electric Polarizability Calculations: Atoms, Molecules and Clusters, edited by G. Maroulis (IOS Press, Amsterdam, 2006) pp. 1-32, http://ctcp.massey.ac.nz/dipole-polarizabilities.

[11] S. Chattopadhyay, B. K. Mani, and D. Angom, Phys. Rev. A 86, 022522 (2012).

[12] S. Chattopadhyay, B. K. Mani, and D. Angom, Phys. Rev. A 86, 062508 (2012). 
[13] S. Chattopadhyay, B. K. Mani, and D. Angom, Phys. Rev. A 87, 042520 (2013).

[14] S. Chattopadhyay, B. K. Mani, and D. Angom, Phys. Rev. A 87, 062504 (2013).

[15] B. K. Sahoo and B. P. Das, Phys. Rev. A 77, 062516 (2008).

[16] R. J. Bartlett and M. Musiał, Rev. Mod. Phys. 79, 291 (2007).

[17] B. K. Mani, K. V. P. Latha, and D. Angom, Phys. Rev. A 80, 062505 (2009).

[18] K. V. P. Latha, D. Angom, B. P. Das, and D. Mukherjee, Phys. Rev. Lett. 103, 083001 (2009).

[19] H. S. Nataraj, B. K. Sahoo, B. P. Das, and D. Mukherjee, Phys. Rev. Lett. 101, 033002 (2008).

[20] R. Pal, M. S. Safronova, W. R. Johnson, A. Derevianko, and S. G. Porsev, Phys. Rev. A 75, 042515 (2007).

[21] G. Gopakumar, H. Merlitz, S. Majumder, R. K. Chaudhuri, B. P. Das, U. S. Mahapatra, and D. Mukherjee, Phys. Rev. A 64, 032502 (2001).

[22] T. A. Isaev, A. N. Petrov, N. S. Mosyagin, A. V. Titov, E. Eliav, and U. Kaldor, Phys. Rev. A 69, 030501 (2004).

[23] G. Hagen, T. Papenbrock, D. J. Dean, and M. HjorthJensen, Phys. Rev. Lett. 101, 092502 (2008).

[24] R. F. Bishop, P. H. Y. Li, D. J. J. Farnell, and C. E. Campbell, Phys. Rev. B 79, 174405 (2009).

[25] A. Derevianko, S. G. Porsev, and K. Beloy, Phys. Rev. A 78, 010503(R) (2008).

[26] P. J. Mohr, B. N. Taylor, and D. B. Newell, Rev. Mod. Phys. 84, 1527 (2012).

[27] G. E. Brown and D. G. Ravenhall, Proc. R. Soc. London, A. 208, 552 (1951).

[28] J. Sucher, Phys. Rev. A 22, 348 (1980).

[29] R. E. Stanton and S. Havriliak, J. Chem. Phys. 81, 1910 (1984).

[30] A. K. Mohanty and E. Clementi, J. Chem. Phys. 93, 1829 (1990).

[31] I. Grant, in Springer Handbook of Atomic, Molecular, and Optical Physics, edited by G. Drake (Springer, New York, 2006) pp. 325-357.

[32] I. P. Grant, Relativistic Quantum Theory of Atoms and Molecules: Theory and Computation (Springer, New York, 2010).

[33] G. D. Purvis and R. J. Bartlett, J. Chem. Phys. 76, 1910
(1982).

[34] I. Shavitt and R. Bartlett, Many-Body Methods in Chemistry and Physics (Cambridge University Press, Cambridge, 2009).

[35] I. Lindgren and J. Morrison, Atomic Many-Body Theory (Springer, Berlin, 2nd Edition, 1986).

[36] S. G. Porsev and A. Derevianko, Phys. Rev. A 73, 012501 (2006).

[37] J. Čížek, Adv. Chem. Phys. 14, 3589 (1969).

[38] R. K. Chaudhuri, P. K. Panda, and B. P. Das, Phys. Rev. A 59, 1187 (1999).

[39] P. Jönsson, G. Gaigalas, J. Bieroń, C. Froese Fischer, and I. P. Grant, Comp. Phys. Comm. 184, 2197 (2013).

[40] C. Froese Fischer, Comp. Phys. Comm. 43, 355 (1987).

[41] P. Pulay, Chem. Phys. Lett. 73, 393 (1980).

[42] J. Mitroy and M. W. J. Bromley, Phys. Rev. A 68, 052714 (2003).

[43] L. Hamonou and A. Hibbert, J. Phys. B 41, 245004 (2008).

[44] Y. Singh, B. K. Sahoo, and B. P. Das, Phys. Rev. A 88, 062504 (2013).

[45] S. Porsev and A. Derevianko, Journal of Experimental and Theoretical Physics 102, 195 (2006).

[46] L. Lundin, B. Engman, J. Hilke, and I. Martinson, Phys. Scr. 8, 274 (1973).

[47] I. S. Lim and P. Schwerdtfeger, Phys. Rev. A 70, 062501 (2004).

[48] A. J. Sadlej, M. Urban, and O. Gropen, Phys. Rev. A 44, 5547 (1991).

[49] T. M. Miller and B. Bederson, Phys. Rev. A 14, 1572 (1976).

[50] H. L. Schwartz, T. M. Miller, and B. Bederson, Phys. Rev. A 10, 1924 (1974).

[51] S. Schäfer, M. Mehring, R. Schäfer, and P. Schwerdtfeger, Phys. Rev. A 76, 052515 (2007).

[52] R. D. Cowan and D. C. Griffin, J. Opt. Soc. Am. 66, 1010 (1976).

[53] B. K. Mani and D. Angom, Phys. Rev. A 81, 042514 (2010).

[54] B. K. Mani and D. Angom, Phys. Rev. A 83, 012501 (2011). 